\title{
OPTIMAL CONTROL FOR THE SUN-POWERED AIRPLANE WITH TAKING INTO ACCOUNT EFFICIENCY OF ONBOARD ACCUMULATOR CHARGING-DISCHARGING AND CHARGE LIMITS
}

\author{
S. V. Serokhvostov ${ }^{1}$ and T. E. Churkina ${ }^{2}$ \\ ${ }^{1}$ Moscow Institute of Physics and Technology \\ Department of Aeromechanics and Flight Engineering \\ 16 Gagarina Str., Zhukovsky 140180, Moscow Region, Russia \\ ${ }^{2}$ Moscow Aviation Institute (National Research University) \\ 4 Volokolamskoye Shosse, Moscow 125993, Russia
}

\begin{abstract}
The problem of optimal control for the aircraft with the electric powerplant and solar cells for the multiday flight is investigated using the more precise equation of motion comparing to the previous investigations. The cases of some restrictions on aircraft energy storage and peculiarities of its charge and discharge are also analyzed. Pontryagin's maximum principle is utilized. Optimal trajectories were obtained for the cases considered.
\end{abstract}

\section{INTRODUCTION}

By this time, a set of solar-powered (SP) airplanes have been designed and built (from those are "Pathfinder," "Centurion," "Helios," "Solar Impuls," "SoLong," "Zephyr," and others). But for the present state-of-the-art, the design of the SP airplane for multiday mission is a serious problem because of the moderate value of the solar radiation intensity, rather low efficiency and rather high density of the solar cells, insufficient energy density of the onboard energy storage, and some other factors.

The performance of the apparatus can be improved through the optimal control in the flight. Some theoretical investigations were made by authors earlier and a set of optimal control problems was solved [1-3]. Hypothesis of "quasistead" motion was used for the equations of motion simplification. Among other results, optimal altitude for the level flight and optimal trajectory for the flight at changing altitude were found analytically.

(C) The Authors, published by EDP Sciences. This is an open access article distributed under the terms of the Creative Commons Attribution License 4.0 (http://creativecommons.org/licenses/by/4.0/). 
But in these investigations, it was assumed that the onboard energy source ("accumulator") has infinite capacity and can accumulate any amount of energy. In fact, any storage device can accumulate only limited amount of energy depending on the storage performance.

Also, the efficiencies of "accumulator" charging and discharging were not taken into account. It is evident that these efficiencies affect the shape of trajectory.

Considered here is the problem of optimal control for the SP airplane for the maximization the "accumulator" energy at the end of flight for the multiday 24-hour periodical mission taking into account capacity of "accumulator" and charging-discharging efficiency.

The research was made on the basis of the Pontryagin's maximum principle (to be more precise, Pontryagin's minimum principle) [4]. Analyzed is the case for which the dependence of the characteristics of the Sun does not change from day to day. For the present investigation, more precise equations of motion compared to the previous investigations were used.

The solutions were checked for the extremum necessary condition.

\section{PREVIOUS INVESTIGATIONS}

Some questions concerning the optimal multiday flight were investigated in [1-3]. First of all, the optimal altitude for the flight at constant altitude was found. If the dependence of air density $\rho$ on the altitude $h$ is defined, then the values of $\rho$ and $h$ can be found from

$$
\rho^{3}=8\left(\eta \frac{\partial J}{\partial h}\right)^{-2}\left(\frac{G}{S}\right)^{3} \sqrt{\frac{A^{3} C_{D 0}}{27}}\left(\frac{\partial \rho}{\partial h}\right)^{2} .
$$

Here, $\eta$ is the powerplant efficiency (assumed to be constant); $G=m g$ where $m$ is the aircraft mass and $g$ is the acceleration of gravity; $S$ is the wing area; $A=1 /(\pi \lambda)$ where $\lambda$ is the aspect ratio of the wing; $C_{D 0}$ is the drag coefficient at zero lift; and $J$ is the power obtained from the unit area of solar cell.

The equation for the isothermal atmosphere in which the density depends on the altitude by the formula defined in [1] as $\rho=\rho_{0} \exp \left(-h / h_{0}\right)\left(h_{0}=6374 \mathrm{~m}\right.$ and $\rho_{0}$ is the air density at the reference level where $\left.h=0\right)$ gives:

$$
\rho=8\left(\eta h_{0} \frac{\partial J}{\partial h}\right)^{-2}\left(\frac{G}{S}\right)^{3} \sqrt{\frac{A^{3} C_{D 0}}{27}} .
$$

The corresponding velocity $V$ is [1]:

$$
V=-\sqrt{\frac{3}{A C_{D 0}}} \frac{\eta \rho}{2(G / S) \partial \rho / \partial h} \frac{\partial J}{\partial h}
$$


that for the isothermal atmosphere gives:

$$
V=\sqrt{\frac{3}{A C_{D 0}}} \frac{\eta h_{0}}{2(G / S)} \frac{\partial J}{\partial h} .
$$

Then, optimal flight path was investigated for the case of nonfixed altitude with the help of Pontryagin's maximum principle [4]. The problem was investigated numerically [1] and analytically [2]. It was found that the hypothesis of quasi-stationary flight can be used for such investigation and within this hypothesis, the flight altitude and velocity are defined by the same relationship as (1) and (2) and the only difference is that the characteristics of Sun radiation are not mean values but the values at the required moment of time. But during the night time, there is no Sun radiation and the optimal air density is equal to infinity (corresponding altitude is equal to "minus infinity"). So, during the night time, the aircraft must fly at the lowest possible altitude.

\section{IMPROVED MODEL OF FLIGHT MECHANICS}

In previous investigations, the following system of governing differential equations of motions was used for numerical investigations [1]:

$$
\left.\begin{array}{c}
m \dot{V}=\frac{W \eta}{V}-\left(C_{D 0}+A C_{L}^{2}\right) \rho \frac{V^{2}}{2} S-m g \sin \theta ; \\
m V \dot{\theta}=C_{L} \rho \frac{V^{2}}{2} S-m g \cos \theta ; \\
\dot{h}=V \sin \theta ; \quad \dot{E}=W-I(h, t)
\end{array}\right\}
$$

where $W$ is the power consumed by the engine $\left(W_{\min }<W<W_{\max }\right) ; C_{L}$ is the lift coefficient; $\theta$ is the angle of trajectory inclination; $E$ is the energy consumed from accumulators; $I=J S$; and $t$ is the time of the day.

According to the "quasi-stead" motion hypothesis, they were simplified in [13] to the form of

$$
\begin{gathered}
m \dot{V}=\frac{W \eta}{V}-\left(C_{D 0}+A C_{L}^{2}\right) \rho \frac{V^{2}}{2} S ; \\
\dot{E}=W-I(h(\rho), t) ; \quad C_{L} \rho \frac{V^{2}}{2} S=m g .
\end{gathered}
$$

In the present investigation we propose the simplification of system (3) to the form

$$
\left.\begin{array}{c}
m \dot{V} V=W \eta-\left(C_{D 0}+A C_{Y}^{2}\right) \rho \frac{V^{3}}{2} S-m g V_{Y} \\
\dot{h}=V_{Y}=V \sin \theta ; \quad \dot{E}=W-I(h, t) ; \quad C_{L} \rho \frac{V^{2}}{2} S=m g
\end{array}\right\}
$$


where $V_{Y}\left(V_{Y \text { min }}<V_{Y}<V_{Y \text { max }}\right)$ is the vertical component of the aircraft velocity. System (4) is more precise than the previous one. Let us introduce new variable:

$$
Z=V^{2}
$$

So, the first equation becomes:

$$
\frac{m}{2} \dot{Z}=W \eta-\left(C_{D 0}+A\left(\frac{2 m g}{\rho Z}\right)^{2}\right) \rho \frac{Z^{3 / 2}}{2} S-m g V_{Y}
$$

The optimized function is the energy $E$ consumed from accumulators during $24 \mathrm{~h}(T=24 \mathrm{~h})$ of flight:

$$
E=\int_{0}^{T}(W-I(h, t)) d t
$$

with the condition that the solution is 24 -hour periodic. Minimum of $E$ is required.

Optimization problem for Pontryagin's minimum principle is analyzed. For this problem, the Hamilton function $\mathbf{H}$ for control variables $W$ and $V_{Y}$ is:

$$
\begin{aligned}
\mathbf{H}=\frac{2 P_{Z}}{m}\left(W \eta-\left(C_{D 0}+A\left(\frac{2 m g}{\rho Z S}\right)^{2}\right) \rho \frac{Z^{3 / 2}}{2}\right. & \left.-m g V_{Y}\right) \\
& +P_{H} V_{Y}+(W-I(h, t))
\end{aligned}
$$

where $P_{Z}$ and $P_{H}$ are the conjugate variables for $Z$ and $h$, respectively.

The conditions for the conjugate variables are:

$$
\begin{aligned}
& \dot{P}_{Z}=-\frac{\partial \mathbf{H}}{\partial Z}=-\left(-\frac{2 P_{Z}}{m}\left(\frac{3}{2} C_{D 0} \rho \frac{Z^{1 / 2}}{2} S-\frac{1}{2} \frac{A(2 m g)^{2}}{2 Z^{3 / 2} \rho S}\right)\right) \\
& \dot{P}_{H}=-\frac{\partial \mathbf{H}}{\partial h}=-\left(-\frac{2 P_{Z}}{m}\left(C_{D 0} \frac{Z^{3 / 2}}{2} S \frac{\partial \rho}{\partial h}-\frac{A(2 m g)^{2}}{2 Z^{1 / 2} \rho^{2} S} \frac{\partial \rho}{\partial h}\right)+\left(-\frac{\partial I}{\partial h}\right)\right) .
\end{aligned}
$$

Function $\mathbf{H}$ is linear with respect to $W$ and $V_{Y}$; so,

- for $W$ :

$$
\text { - } W= \begin{cases}W_{\max } & \text { at } s<0 \\ W_{\min } & \text { at } s>0\end{cases}
$$

- singular control: $s=0, \dot{s}=0$ 
where $s$ is the switch function

$$
s=\frac{2 \eta P_{Z}}{m}+1
$$

- for $V_{Y}$ :

- $V_{Y}= \begin{cases}V_{Y \max } & \text { at } p<0 ; \\ V_{Y \min } & \text { at } p>0 ;\end{cases}$

- singular control $p=0, \dot{p}=0$

where $p$ is the switch function

$$
p=-2 P_{Z} g+P_{H} .
$$

Singular control of $W$ gives:

$$
s=\frac{2 \eta P_{Z}}{m}+1=0 ; \quad \dot{s}=\frac{2 \eta \dot{P}_{Z}}{m}=0 .
$$

Taking into account (6), one can obtain:

$$
\dot{s}=\frac{2 \eta}{m} \frac{2 P_{Z}}{m}\left(\frac{3}{2} C_{D 0} \rho \frac{Z^{1 / 2}}{2} S-\frac{1}{2} \frac{A(2 m g)^{2}}{2 Z^{3 / 2} \rho S}\right)=0 .
$$

From (8), $P_{Z} \neq 0$; so,

$$
\frac{3}{2} C_{D 0} \rho \frac{Z^{1 / 2}}{2} S-\frac{1}{2} \frac{A(2 m g)^{2}}{2 Z^{3 / 2} \rho S}=0 .
$$

Together with

$$
C_{L} \rho \frac{V^{2}}{2} S=m g
$$

it gives the conditions:

$$
\rho Z=\text { const } ; \quad A C_{Y}^{2}=3 C_{D 0} .
$$

Singular control condition for $V_{Y}$ (9) with (6) and (7) gives:

$$
\dot{P}_{H}=-\frac{1}{\eta}\left(C_{D 0} \frac{Z^{3 / 2}}{2} S \frac{\partial \rho}{\partial h}-\frac{A(2 m g)^{2}}{2 Z^{1 / 2} \rho^{2} S} \frac{\partial \rho}{\partial h}\right)+\left(\frac{\partial I}{\partial h}\right)=0 .
$$

Two these conditions of singular control, (10) and (11) lead to Eq. (1). So, it was proved that the previously obtained conditions of optimal flight are valid also in this improved model of flight. 
Then, it is required to check the solution for the necessary extremum condition [4]:

$$
\begin{gathered}
-\frac{\partial}{\partial W}\left(\frac{d^{2}}{d t^{2}}\left(\frac{\partial \mathbf{H}}{\partial W}\right)\right) \geq 0 \\
-\frac{\partial}{\partial V_{Y}}\left(\frac{d^{2}}{d t^{2}}\left(\frac{\partial \mathbf{H}}{\partial V_{Y}}\right)\right) \geq 0
\end{gathered}
$$

with taking into account the equations obtained above.

For the first condition,

$$
\begin{aligned}
& \frac{d^{2}}{d t^{2}}\left(\frac{\partial \mathbf{H}}{\partial W}\right)=\frac{d \dot{s}}{d t}=\frac{d}{d t}\left(\frac{2 \eta \dot{P}_{Z}}{m}\right) \\
&=\frac{d}{d t}\left(\frac{2 P_{Z}}{m}\left(\frac{3}{2} C_{D 0} \rho \frac{Z^{1 / 2}}{2} S-\frac{1}{2} \frac{A(2 m g)^{2}}{2 Z^{3 / 2} \rho S}\right)\right) \\
&=\frac{2 \dot{P}_{Z}}{m}\left(\frac{3}{2} C_{D 0} \rho \frac{Z^{1 / 2}}{2} S-\frac{1}{2} \frac{A(2 m g)^{2}}{2 Z^{3 / 2} \rho S}\right) \\
& \quad+\frac{2 P_{Z}}{m} \frac{d}{d t}\left(\frac{3}{2} C_{D 0} \rho \frac{Z^{1 / 2}}{2} S-\frac{1}{2} \frac{A(2 m g)^{2}}{2 Z^{3 / 2} \rho S}\right) .
\end{aligned}
$$

As for the singular control (8) $\dot{P}_{Z}=0$, then

$$
\begin{array}{r}
\frac{d^{2}}{d t^{2}}\left(\frac{\partial \mathbf{H}}{\partial W}\right)=\frac{2 P_{Z}}{m} \frac{d}{d t}\left(\frac{3}{2} C_{D 0} \rho \frac{Z^{1 / 2}}{2} S-\frac{1}{2} \frac{A(2 m g)^{2}}{2 Z^{3 / 2} \rho S}\right) \\
=\frac{2 P_{Z}}{m}\left(\frac{3}{2} C_{D 0} \rho \frac{\dot{Z}}{4 Z^{1 / 2}}+\frac{3}{2} \cdot \frac{1}{2} \frac{A(2 m g)^{2} \dot{Z}}{2 Z^{5 / 2} \rho S}+\frac{3}{2} C_{D 0} \frac{\partial \rho}{\partial h} \frac{Z^{1 / 2}}{2} S \dot{h}\right. \\
\left.+\frac{1}{2} \frac{A(2 m g)^{2}}{2 Z^{3 / 2} \rho^{2} S} \frac{\partial \rho}{\partial h} \dot{h}\right) .
\end{array}
$$

Taking into account (5), one can obtain:

$$
\frac{\partial}{\partial W}\left(\frac{d^{2}}{d t^{2}}\left(\frac{\partial \mathbf{H}}{\partial W}\right)\right)=\left(\frac{2}{m}\right)^{2} P_{Z}\left(\frac{3}{2} C_{D 0} \rho \frac{1}{4 Z^{1 / 2}}+\frac{3}{2} \cdot \frac{1}{2} \frac{A(2 m g)^{2}}{2 Z^{5 / 2} \rho S}\right) .
$$

Expression in brackets is positive. From (8), $P_{Z}<0$; so,

$$
-\frac{\partial}{\partial W}\left(\frac{d^{2}}{d t^{2}}\left(\frac{\partial \mathbf{H}}{\partial W}\right)\right) \geq 0 \text {. }
$$


For the other condition,

$$
\frac{d^{2}}{d t^{2}}\left(\frac{\partial \mathbf{H}}{\partial V_{Y}}\right)=\frac{d^{2} p}{d t^{2}}=\frac{d}{d t}\left(-2 \dot{P}_{Z} g+\dot{P}_{H}\right)
$$

From this,

$$
\begin{aligned}
\frac{d}{d t}\left(-2 \dot{P}_{Z} g+\dot{P}_{H}\right)= & \frac{d}{d t}\left(\frac{2 P_{Z}}{m}\left(C_{D 0} \frac{Z^{3 / 2}}{2} S \frac{\partial \rho}{\partial h}-\frac{A(2 m g)^{2}}{2 Z^{1 / 2} \rho^{2} S} \frac{\partial \rho}{\partial h}\right)\right. \\
& \left.+\frac{\partial I}{\partial h}-2 g \frac{2 P_{Z}}{m}\left(\frac{3}{2} C_{D 0} \rho \frac{Z^{1 / 2}}{2} S-\frac{1}{2} \frac{A(2 m g)^{2}}{2 Z^{3 / 2} \rho S}\right)\right) .
\end{aligned}
$$

For the isothermal atmosphere (11-20 km above the Earth), as it was mentioned above, $\rho=\rho_{0} \exp \left(-h / h_{0}\right)$. From this,

$$
\frac{\partial \rho}{\partial h}=-\frac{\rho}{h_{0}} .
$$

As $\dot{P}_{Z}=0$ and taking into account (8),

$$
\begin{aligned}
\frac{d^{2}}{d t^{2}}\left(\frac{\partial \mathbf{H}}{\partial V_{Y}}\right)=\left(\frac{2 P_{Z}}{m}\right. & \left(C_{D 0} \frac{Z^{3 / 2}}{2} S \frac{\rho}{h_{0}^{2}}+\frac{A(2 m g)^{2}}{2 Z^{1 / 2} \rho h_{0}^{2} S}\right)+\frac{\partial^{2} I}{\partial h^{2}} \\
& \left.+2 g \frac{2 P_{Z}}{m}\left(\frac{3}{2} C_{D 0} \rho \frac{Z^{1 / 2}}{2 h_{0}} S+\frac{A(2 m g)^{2}}{2 Z^{3 / 2} \rho h_{0} S}\right)\right) V_{Y}+\cdots
\end{aligned}
$$

For the atmosphere with the density decreasing with altitude, the corresponding graphs are the convex upwards (Fig. 1); so,

$$
\frac{\partial^{2} I}{\partial h^{2}}<0
$$

and, as $P_{Z}<0$, the expression in brackets is negative.

From this,

$$
-\frac{\partial}{\partial V_{Y}}\left(\frac{d^{2}}{d t^{2}}\left(\frac{\partial \mathbf{H}}{\partial V_{Y}}\right)\right)>0 .
$$

So, it is proved that the necessary condition for the singular control is fulfilled.

As an example, let us consider some generic aircraft with the following characteristics: $C_{D 0}=0.01 ; \lambda=30 ; m=600 \mathrm{~kg} ; S=200 \mathrm{~m}^{2} ; \eta=0.8$; and solar cell efficiency $23.2 \%$. Taking the mean value of data from $[5,6]$, one can obtain the average value of $\partial J / \partial h$ for $24 \mathrm{~h}$ as $\partial J / \partial h=0.0025 \mathrm{~W} / \mathrm{m}^{3}$. These values give the "constant optimal altitude" (COA) of about $9 \mathrm{~km}$. 


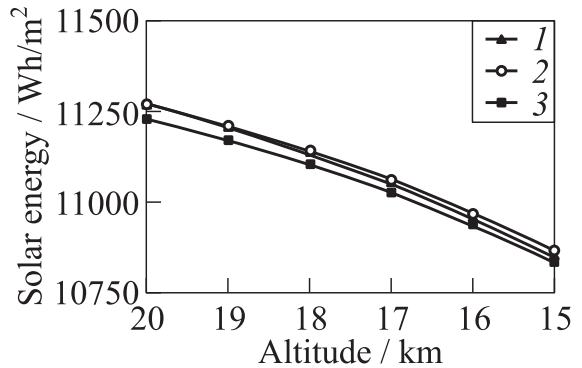

(a)

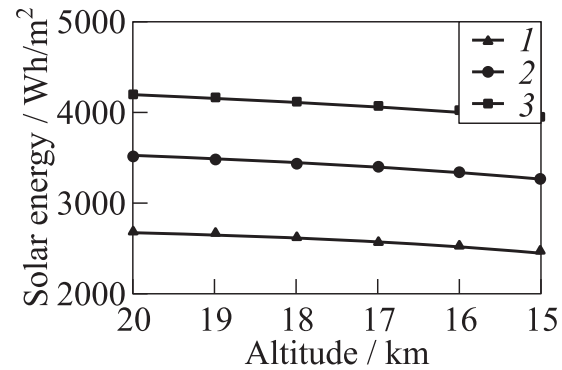

(b)

Figure 1 Dependence of solar energy on altitude [5] on June $22(a)$ and on December $22(b): 1-L=45^{\circ} ; 2-40^{\circ}$; and $3-L=36^{\circ}$

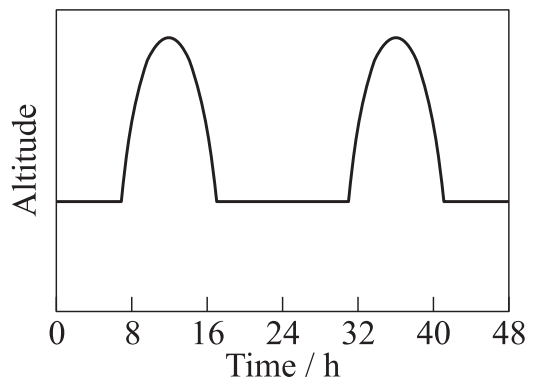

Figure 2 Proposed trajectory

According to formula (1) used for instant density (and altitude) during the flight at variable altitude, at night the density (and the altitude) is equal to "minus infinity" (as was shown in [1]). So, during the night, the aircraft must fly at some altitude restriction. Let us consider the situation proposed in [1]: the minimal altitude is the COA. So, during the day time, for rather high values of $\partial J / \partial h$, the altitude is higher than COA; for the night time and during the sunrise and sunset, the altitude is equal

to COA (Fig. 2).

For this trajectory, the advantage in energy stored for $24 \mathrm{~h}$ (compared to the flight at COA) is about $30 \%$ of the energy consumed by motors for the 24 -hour flight at $\mathrm{COA}$.

The maximal altitude difference is about $2.3 h_{0}(14650 \mathrm{~m})$ and is independent of other parameters of aircraft and atmosphere.

Of cause, one can propose another altitude limitations.

\section{INFLUENCE OF CHARGE-DISCHARGE EFFICIENCY}

Formula (1) gives the dependency of the altitude shape on the efficiency of powerplant as a whole and, in particular, on the charge-discharge efficiency. One can 
see that the lower the efficiency, the higher the air density, or, in other words, the lower the altitude. But we are to investigate this question more thoroughly.

Assume that the part of energy $\alpha$ from solar cells goes to the motor and the other part of energy $(1-\alpha)$ goes to accumulator to charge it. Assume that the accumulator charging efficiency is $\gamma$ and discharging efficiency is $1 / \beta, \eta$ here is the efficiency of all the powerplant except accumulator and solar cells. Define that $W$ is the power going from accumulator to the motor; so, $W \geq 0$.

For this investigation, the equations of motion become as follows:

$$
\begin{gathered}
\frac{m}{2} \dot{Z}=(\alpha I(h, t)+W) \eta-\left(C_{D 0}+A\left(\frac{2 m g}{\rho Z}\right)^{2}\right) \rho \frac{Z^{3 / 2}}{2} S-m g V_{Y} \\
\dot{E}=\beta W-\gamma(1-\alpha) I(h, t) .
\end{gathered}
$$

Hamilton function for control variables $\alpha, W$, and $V_{Y}$ is:

$$
\begin{aligned}
\mathbf{H}=\frac{2 P_{Z}}{m}\left((\alpha I(h, t)+W) \eta-\left(C_{D 0}+A\left(\frac{2 m g}{\rho Z S}\right)^{2}\right) \rho \frac{Z^{3 / 2}}{2} S-m g V_{Y}\right) \\
+P_{H} V_{Y}+(\beta W-\gamma(1-\alpha) I(h, t)), \quad 0<\alpha<1,0<W<W_{\max }
\end{aligned}
$$

For the control variables:

- for $W$ :

- $W= \begin{cases}W_{\max } & \text { at } s<0 ; \\ W_{\min }(W=0) & \text { at } s>0 ;\end{cases}$

- singular control: $s=0, \dot{s}=0$

where $s$ is the switch function:

$$
s=\frac{2 \eta P_{Z}}{m}+\beta
$$

- for $\alpha$ :

- $\alpha= \begin{cases}\alpha_{\max } & \text { at } q<0 ; \\ \alpha_{\min }(\alpha=0) & \text { at } q>0 ;\end{cases}$

- singular control: $q=0, \dot{q}=0$

where $q$ is the switch function:

$$
q=\frac{2 \eta P_{Z}}{m}+\gamma
$$


- for $V_{Y}$ :

- $V_{Y}= \begin{cases}V_{Y \max } & \text { at } p<0 ; \\ V_{Y \min } & \text { at } p>0 ;\end{cases}$

- singular control $p=0, \dot{p}=0$

where $p$ is the switch function:

$$
p=-2 P_{Z} g+P_{H}
$$

Consider the cases of singular control. It is evident that the cases of singular controls for $\alpha$ and $W$ does not realize simultaneously as $\beta>1$ and $\gamma<1$ (see (12) and (13)). This means that there are the following situations.

1. Singular control for $\alpha$. As $\gamma<\beta$, then switch function for $W$ is positive. It means that $W$ must be minimal (equal to zero in this case). So, the situation is that a part of power from solar cells goes to the motor, another part goes to accumulator, and no power from accumulator goes to motor.

2. Singular control for $W$. In this case, switch function for $\alpha$ is negative and $\alpha$ must be maximal $(\alpha=1)$. This means that all the power from solar cell goes to the motor and also some power from accumulator goes to the motor.

From this, one can understand that the accumulator cannot charge and discharge simultaneously. On the one hand, it is rather evident but on the other hand, for the accuracy, even evident things must be proved.

For each of singular control cases $s=0$ or $q=0$, the conditions of singular control $\dot{s}=0$ or $\dot{q}=0$ give the same equation:

$$
\dot{P}_{Z}=0
$$

as in previous section.

The condition for the conjugate variable $P_{Z}$ is the same as (6).

The condition for $P_{H}$ is:

$$
\begin{aligned}
& \dot{P}_{H}=-\frac{\partial \mathbf{H}}{\partial h} \\
= & -\left(\frac{2 P_{Z}}{m}\left(\alpha \frac{\partial I}{\partial h}-C_{D 0} \frac{Z^{3 / 2}}{2} S \frac{\partial \rho}{\partial h}-\frac{A(2 m g)^{2}}{2 Z^{1 / 2} \rho^{2} S} \frac{\partial \rho}{\partial h}\right)+\gamma(1-\alpha)\left(-\frac{\partial I}{\partial h}\right)\right) .
\end{aligned}
$$

One can check that for any of singular controls for $\alpha$ or $W$, this equation becomes (7) if one substitutes $\gamma(\partial I / \partial h)$ in place of $(\partial I / \partial h)$. This means that the solutions for $V$ and $h$ are the same as in previous section or, in other words, are given by (1) 
and (2) with the substitution $\gamma(\partial I / \partial h)$ in place of $(\partial I / \partial h)$. If one introduces the "total" powerplant efficiency $\eta_{0}$ with taking into account accumulator charging efficiency as

$$
\eta_{0}=\gamma \eta
$$

one obtains the condition for the altitude in the same form as (1) with substitution $\eta_{0}$ in place of $\eta$.

Formula (1) for the isothermal atmosphere gives the dependency of altitude on the flight parameters as

$$
\frac{h}{h_{0}}=2 \ln \eta_{0}+2 \ln \left(\frac{\partial J}{\partial h}\right)-3 \ln \left(\frac{G}{S}\right)-0.5 \ln \left(\frac{A^{3} C_{D)}}{27}\right)-3 \ln 2+2 \ln \left(h_{0}\right) .
$$

If one assumes that $(\partial J / \partial h)$ is nearly the same at any altitude for the fixed moment of time, then the optimal trajectories for the different values of $\eta_{0}$ are simply shifted in vertical directions but have the same shape. To estimate this shift, one can say that $1 \%$ of efficiency increase is equal to the altitude increase by $127.4 \mathrm{~m}$.

It must be noted that there is no symbol $\beta$ in the results of this section. This means that the discharge efficiency does not influence the altitude and velocity. It influences only on power consumption from accumulators. This acts only during the accumulator energy consumption (at night and in the case of insufficient solar energy).

So, for any singular control, the required power depends only on the time of the day for the flight within the restrictions on the altitude. The only question is what is the source of this energy (solar cell or accumulator).

\section{INFLUENCE OF ONBOARD ENERGY LIMITATION}

It is evident that the energy stored in onboard accumulators cannot be less than zero (fully discharged accumulator) and higher than some maximal amount of energy (fully charged accumulator). Mathematically, these conditions can be expressed as:

$$
\begin{aligned}
& \Phi_{1}=E-E_{\max } \leq 0 \text { (full discharege) } \\
& \Phi_{2}=-E \leq 0 \text { (full charge) }
\end{aligned}
$$

As there is no control variables in this expressions, one must use the derivatives of these equations for Pontryagin's minimum principle [4]. 
For this task, Hamilton function from previous section must be rewritten as:

$$
\begin{aligned}
& \mathbf{H}=\frac{2 P_{Z}}{m}\left((\alpha I(h, t)+W) \eta-\left(C_{D 0}+A\left(\frac{2 m g}{\rho Z S}\right)^{2}\right) \rho \frac{Z^{3 / 2}}{2} S-m g V_{Y}\right)+P_{H} V_{Y} \\
& +(\beta W-\gamma(1-\alpha) I(h, t))+\mu_{1}(\beta W-\gamma(1-\alpha) I(h, t))+\mu_{2}(\beta W-\gamma(1-\alpha) I(h, t)) .
\end{aligned}
$$

Here, $\mu_{1}(t) \geq 0$ for the full charge restriction and $\mu_{2}(t) \leq 0$ for the full discharge restriction; $\mu_{1}(t)=0$ and $\mu_{2}(t)=0$ inside restrictions. It is evident that $\mu_{1}$ and $\mu_{2}$ cannot be higher than zero simultaneously.

One can check that inside the restrictions, the solutions are the same as in the previous section (as in this case $\mu_{1}(t)=0$ and $\mu_{2}(t)=0$ ).

For the case of restrictions,

$$
\dot{E}=\beta W-\gamma(1-\alpha) I(h, t)=0 .
$$

There are two ways to fulfill this dependence:

(1) $W=0$ and $\alpha=1$. In this case, all the energy from solar cells goes to the motor; and

(2) $W>0$ and $\alpha<1$. In this case some energy from solar cells goes to the accumulator and then it goes to motor. In this case, some amount of energy loses on the way from solar cell to the motor comparing to the first case.

So, the first case must be chosen and, thus, the values of two control variables $W$ and $\alpha$ are defined.

The condition for $V_{Y}$ control variable is the same as in the previous section and looks as (9).

As for the conjugate variables, the condition for $P_{Z}$ remains the same as (6) and condition for $P_{H}$ will be as:

$$
\begin{array}{r}
\dot{P}_{H}=-\frac{\partial \mathbf{H}}{\partial h}=-\left(\frac{2 P_{Z}}{m}\left(\alpha \eta \frac{\partial I}{\partial h}-\left(C_{D 0} \rho \frac{Z^{3 / 2}}{2} S \frac{\partial \rho}{\partial h}-\frac{A(2 m g)^{2}}{2 Z^{1 / 2} \rho^{2} S} \frac{\partial \rho}{\partial h}\right)\right)\right. \\
\left.-\gamma(1-\alpha)\left(1+\mu_{1}+\mu_{2}\right)\left(\frac{\partial I}{\partial h}\right)\right) .
\end{array}
$$

As $\alpha=1$, then

$$
\dot{P}_{H}=-\frac{\partial \mathbf{H}}{\partial h}=-\left(\frac{2 P_{Z}}{m}\left(\eta \frac{\partial I}{\partial h}-\left(C_{D 0} \rho \frac{Z^{3 / 2}}{2} S \frac{\partial \rho}{\partial h}-\frac{A(2 m g)^{2}}{2 Z^{1 / 2} \rho^{2} S} \frac{\partial \rho}{\partial h}\right)\right)\right) .
$$

This equation together with (6) and derivative of (9) with respect to time gives:

$$
\begin{gathered}
\left(C_{D 0} \rho \frac{Z^{3}}{2} S \frac{\partial \rho}{\partial h}-\frac{A(2 m g)^{2}}{2 \rho^{2} S} Z \frac{\partial \rho}{\partial h}\right)-\eta \frac{\partial I}{\partial h}-2 g\left(\frac{3}{2} C_{D 0} \rho \frac{Z^{2}}{2} S-\frac{1}{2} \frac{A(2 m g)^{2}}{2 \rho S}\right) \\
=0
\end{gathered}
$$


The equations of motion on the limitations investigated are:

$$
\frac{m}{2} \dot{Z}=I(h, t) \eta-\left(C_{D 0}+A\left(\frac{2 m g}{\rho(h) Z}\right)^{2}\right) \rho(h) \frac{Z^{3 / 2}}{2} S-m g V_{Y} ; \quad \dot{h}=V_{Y} .
$$

Last three equations give the solution for $h$ and $Z$ as function of time.

As an example, let us consider the case with the same conditions as in section 3 and imagine that the accumulators become full at the moment when the aircraft reached the COA in the "evening." The calculations give the trajectory of airplane after this moment as shown in Fig. 3 (dotted line) up to the next moment of reaching the COA.

Investigation of this trajectory

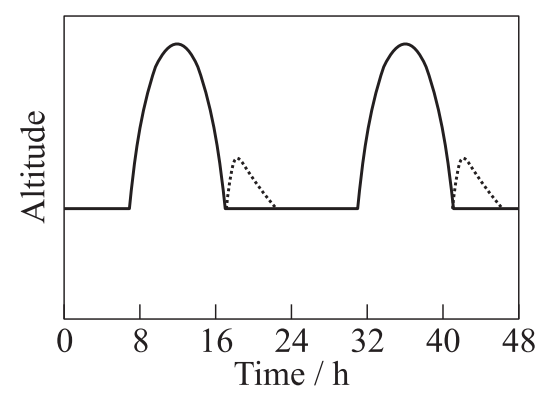

Figure 3 Trajectory for the case with restriction on the accumulator capacity (dotted line) shows that the disadvantage of the stored energy for $24 \mathrm{~h}$ (compared to the case without the restriction on the accumulator capacity) is about $2 \%$ of the energy consumed by the motors for $24 \mathrm{~h}$.

\section{DISCUSSION AND FUTURE WORK}

The solutions obtained above give the optimal control and trajectory for the parts of trajectory inside the restrictions and on the restrictions. Next step must be to join the pieces into the whole trajectory. One of the difficulties to make it using Pontryagin's maximum principle is that the conjugate variables have the discontinuity in the points of connection. So, this problem requires separate investigation.

Also, the assumption of the powerplant efficiency independence of flight conditions and power was made. The investigation for the real powerplant efficiency is also requires in the future.

\section{CONCLUDING REMARKS}

1. Investigations for the optimal control of Sun-powered aircraft for the set of tasks in more precise definition comparing to the previous investigations were conducted and the solutions were obtained. 
2. Influence of the powerplant efficiency and efficiency of charge and discharge was investigated. It was found that the powerplant efficiency and accumulator charging efficiency affect on the optimal trajectory. In particular, the lower the efficiencies, the lower is the altitude. The accumulator discharge efficiency does not affect the trajectory, it affects only the power consumption.

3. The case of energy storage limitations was investigated. The directions of the energy flows were determined and the equations for the trajectory determination were obtained.

\section{REFERENCES}

1. Serokhvostov, S., and T. Churkina. 2007. Optimal control for the Sun-powered airplane in a multi-day mission. 2nd European Conference for Aerospace Sciences. CD.

2. Serokhvostov, S. V., and T. E. Churkina. 2008. Optimal control for the Sun-powered airplane in a multi-day mission. Scientific Proceedings of Riga Technical University. Ser. 6: Transport and engineering. Transport. Aviation transport. 27:212-220.

3. Serokhvostov, S. 2013. Some results of electrical aircraft flight path optimization with the help of Pontryagin maximum principle and their implementation to design optimization. 1st Workshop (International) "Extremal and Record-Breaking Flights of the UAVs and the Aircraft with Electrical Power Plant." Moscow-Ramenskoe.

4. Bryson, A. E., and Y.C. Ho. 1969. Applied optimal control: Optimization, estimation, and control. Waltham, MA: Blaisdell. 481 p.

5. Romeo, G., G. Frulla, and L. Fattore. 2000. HELIPLAT: A solar powered HALEUAV for telecommunication applications. Design and parametric results. Analysis, manufacturing and testing of advanced composite structures. Technical Conference (International) on Uninhabited Aerial Vehicles. Paris, France.

6. Hall, D. W., C. D. Fortenbach, E. V. Dimicelli, and R. W. Parks. 1983. A preliminary study of solar powered aircraft and associated power trains. Technical Report NASACR-3699. 206 p. 